\title{
A Research of Traffic Prediction using Deep Learning Techniques
}

\author{
B.Karthika, N.UmaMaheswari, R.Venkatesh
}

\begin{abstract}
Traffic data is very important in designing a smart city. Now - a day'smany intelligent transport systems use modern technologies to predict traffic flow, to minimize accidents on road, to predict speed of a vehicle and etc. The traffic flow prediction is an appealing study field. Many techniques of data mining are employed to forecast traffic. Deep learning techniques can be used with technological progress to prevent information from real time. Deep algorithms are discussed to forecast real-world traffic data. When traffic data becomes big data, some techniques to improve the accuracy of trafficprediction are also discussed.

Keywords: Deep learning, Neural network, Traffic flow prediction, Convolutional Neural Network (CNN), Recurrent Neural Network (RNN), Stacked Autoencoder (SAE).
\end{abstract}

\section{INTRODUCTION}

Transportation uses recent digital techniques to achieve efficient traffic flow, minimize accidents on road and maintain speed on road, Traffic predictions helps us in route planning, navigation, and other mobilityservices. Data traffic are real world information, i.e. traffic models are usually used to evaluate different past and real-time trafficdata to forecast potential traffic circumstances. speed are loop detectors, trafficcameras, and radars and so on. They need high maintenance and deployment cost. Hence the only a small range is covered using such high cost infrastructures. With the help of smart phones, GPS devices, sensors, and etc, tracking of vehicle can be done with in small amount of time. Modern technologies use data mining algorithm to find prediction in transportation field. Once the data becomes more and more, data mining algorithm performance will decrease. So, machine learning and deep learning techniques can be used to predict data accurately.

To elegantly design a smart city, smart transportation also plays a major role. In smart transportation design, traffic forecasting has many purposes for example to calculate capacity of infrastructure, to calculate air pollution and noise level and etc. Deep learning is employed because it automatically extracts features from large set of data. Deep learning approach use multi layer to find hidden information in transaction.

Revised Manuscript Received on July 18, 2019.

Mrs.B.Karthika, Assistant professor, Dept of Information Technology,PSNA college of engineering and Technology, Dindigul, Tamilnadu, India.(email: karthika@ psnacet.edu.in)

Dr.N.UmaMaheswari, Professor,Dept of Computer science and Engineering,PSNA college of engineering and Technology, Dindigul, Tamilnadu, India.(email: numamahi@gmail.com)

Dr.R.Venkatesh, Professor,Dept of Information Technology,PSNA college of engineering and Technology, Dindigul, Tamilnadu, India.

(email: rvenkatit@ psnacet.edu.in)
So, infrastructures are needed to monitor traffic flow,

\section{LITERATURE REVIEW}

The short-term traffic forecast is essential for the development of intelligent transport systems (ITSs), where we are and where we are going online is the proposed author's short-term traffic forecast. A lot of solutions to traffic features and traffic situations have previously been followed. Recent technological development of machine learning can be monitored and traffic forecasts can be improved [1].

In Traffic Flow Prediction, big data: The traffic flow data provided by the author of a deep learning approach is essential for the effective functioning of intelligent transport systems. It doesn't fit many applications in the real world. A layered auto encoder design for predicting traffic situations is used in a deep learning approach. comparison of other data mining models has demonstrated in an efficient way.[2]

A transport traffic estimation technique has been introduced by the Mining Road Network Correlation for traffic estimation through the author of Compressive Sensing. This strategy has been tested with traffic data of over 4400 taxis from Shanghai town, China. The author suggests a strategy to estimating traffic that reduces the effort of people and enhances automation. Accuracy estimates are used to validate this method [4]

In the Citywide Crowd Flows Deep Spatio-Temporal Residual Networks, crowds are expected to move. They play an important part in traffic management. Many factors such as events, weather etc. affect it. They suggested a profound deep learning strategy, known as ST-ResNet, to forecast crowds in all regions of a town. The results of the three remaining neural networks can be aggregated on the basis of data and are assigned to various branches and regions by ST-ResNet. Two kinds of crowd-flows are experimented with in Beijing and New York City.[5]

In the Real-Time Crash Prediction Dynamic Bayesian Network Motor, the data paper has suggested the Bayesian network dynamic (DBN) system. DBN systems in Shanghai, China were constructed with a time series for the real-time research of 551 accidents and related velocity data, gathered on expressways. A comparison assessment of the model DBN and other machine learning algorithms was also carried out [6].

In Learning Traffic as Image: A Large-Scaled

Published By: 
Transportation Network Deep Convolutional Neural Network Speed Prediction Author proposed a technique of learning traffic as images based on a Convolutionary Neural Network (CNN). A CNN is used in the image in two successive phases: explicit traffic removal function and networkwide estimation of traffic speed. It evaluates by using real-world data and compares it to three deep-learning layered-auto-encoders, RNN and LTMN that is, ordinary least squares, k-nearest neighbors, artificial neural network and random forests. This approach is used in comparison to such algorithms. [7]

Speed prediction in traffic and Source exploration of congestion: a deep learning method suggested LC-RNN for the precise technology of congestion forecast. The RNN and CNN models are integrated. In fact, it is proposed that a network built-in convolution model identifies road characteristics.[9]

In a predictive traffic forecast using a Deep Learning Paradigm, artificial neural network concepts are provided for prediction, with the help of 2 real datasets using suggested LC-RNN algorithms. Intelligent Transport Systems (ITS) collect and process enormous quantities of information from a range of sensors for many years. A forecast is not one of these two real datasets. It exceeds other teaching modules on the device. [17].

Deep learning for road traffic trials is intended to manage Floating Car Data (FCD) historical speeds to estimate road traffic data and to estimate how much progress depends on the architecture (FNN, CNN and LSTM) and to specify the statistics. A RMSE precision metric is assessed against the suggested profound learning architecture. 18] Deep Learning theory forecast methods for traffic data were described in the Large-Scale Transportation Network Congestion Evolution Prediction. In the large transportation network, it is essential to understand congestion in order to prevent traffic delays. For the forecast of congestion, traditional techniques use static information. Transport data is becoming increasingly ubiquitous with new techniques such as Intelligent Transportation Systems (ITS) and the Internet of Things (IoT). To forecast traffic congestion, we present a profound restricted Boltzmann machine and the recurrent neural network design. Compared with current machine learning algorithms, the suggested architecture predicts traffic data more exactly and precisely. The prediction of traffic data is a challenge owing to short-term (e.g. accidents, constructions) and long-term (e.g. peak, hour, seasonal or weather) pattern. The projection of shortterm traffic data is the result of the architecture of long short-term memory (LSTM), used to predicting traffic data, which is the long cycle of the LSTM architecture, [10] which is a generic approach in deep learning. It works well against other current algorithms of machine learning. [11] A Deep Transport concept for traffic congestion was presented in Citywide Level Prediction and Simulation of Human Mobility and Transportation. The traffic caused significant financial lack of fuel, excessive air pollution, time lost and productivity reduction. The system's effectiveness has been validated by experimental outcomes, which are Deep Transportation, to simulate and to predict human mobility. The method of human transport on is also predictable. 12][ 13][ 23]

\section{DEEP LEARNING BASED TRAFFIC PREDICTION ALGORITHMS \& RESULTS}

Although different types of deep learning models occur, they share a popular architecture that includes an input layer, output layer and between several and over 1000 hidden levels. Raw data initializes the input layer characteristics, while the output layer issues the required inferences. All hidden layers can convert the input layer states into the anticipated result of the outcome layer by recording high-level abstractions. The number of devices in each location on the network can change and the dimensions of the network can differ. There are links across systems of any two adjacent levels and each connection has a weight. Each device has an active function that determines how its own status can be calculated on the basis of measurements from the instant predecessor. The rectified linear device (ReLU) is recently one of the most populous activation functions and consists of a half-wave rectifier $\mathrm{f}(\mathrm{x})=$ $\max (\mathrm{x}, 0) \cdot[19]$

\section{i. Convolutional Neural Network (CNN):}

The CNN model is intended mainly for processing 2dimensional data, such as images. A CNN model consists of an input layer and an output layer, together with several hidden layers that can be the convolution layers, pooling or fully connected. The convolutionary layers use convolutionary filters to record their characteristics using certain modifications on the input data. The next step is to mix the device cluster output from a prior phase with the max and/or min matrix into a separate device in the next phase. An abstract depiction of the data is learned by a pooling layer, which in the meantime acts as a form that reduces dimensionality to facilitate the whole model.

\section{ii. Recurrent Neural Network (RNN):}

The RNN model is primarily used for sequential input tasks. RNNs method a single component input sequence and maintain performance results on hidden components that contain historical information of all previous components implicitly. If the circuit is unfolded, a RNN can be seen as a pile of distinct neural networks with parameters of each network supplied from the previous one. The input component is linked to the output component of the past phase in the repeating neural networks of a RNN and are then transmitted together into an activating feature (e.g. tan h) for performance derived. This architecture enables RNNs to record the dynamics of time but procedures that do not allow RNNs to sustain the longer-term dependence. Thus, an enhanced RNN is suggested which utilizes unique hidden units (i.e. memory cells, to recall long-term outputs) known as the Long Short-Term Memory Network (LSTM). LSTM models can study lengthy sequences and determine the best prediction time lags automated [19].

\section{iii. Stacked Autoencoder (SAE):}

An autoencoder is an input layer, an output layer, and hidden layer of a three-layer neural network. The destination output is purposely defined as the model input, and the 
hidden element seeks for the representation of the input information to be understood as decrease of dimensionality or input information coding. The hidden part of an autoencoder is also called a function layer because of this function. The SAE model connects these function levels to produce higher-level input data abstractions that generate a profound architecture. One of the most common autoencoders is a derivative autoencoder, which intentionally requires damaged samples while forcing the original information to be recovered. Thereby, we obtain a variant called a stacked denoising autoencoder (SdAE) when various denoising autoencoders are stacked. SdAE is comparatively stable, making it robust against noisy inputs, and therefore performing significantly faster in comparison with the SAE model.

\section{PROPOSED METHOD}

Data are collected from different sources and parameter selection is done. The evaluations of the proposed algorithm against metrics (i.e., RMSE, RMS) areconsidered. Proposed methods use a multimodal architecture for data collection. A prediction is implemented to each data source using a deep learning algorithm. All predictions are fused together to obtain result. Therefore, the prediction of multimodal traffic is attractive where ensemble learning theory is integrated to produce a better result. A number of deep learning models for various forms of mobility to acquire various traffic models and to implement a weighted average approach. It analyzes traffic data using a hybrid of current algorithms. [19]

Parallel computing may be carried out to enhance the effectiveness of the prediction technique. Knowing how to parallel the work to the entire final model for fast prediction remains and to combine the pieces of parameters studied at distinct machines is essential. In the PS architecture, the server nodes maintain the latest model parameters and provide them to working nodes while worker nodes update model parameters with the assigned data for training purposes. The PS parameter server (PS) system is used for the management and synchronization of machine model parameters.

\section{CONCLUSION}

We discuss the rich mobility data and deep learning about urban traffic predictions. Deep learning promotes traffic predictions through powerful fair representation learning.

\section{REFERENCES}

[1] E. I. Vlahogianni, M. G. Karlaftis, and J. C. Golias, "Short-Term Traffic Forecasting: Where We Are and Where We'reGoing," Transportation Research Part C: Emerging Technologies, vol. 43, part 1, 2014, pp. 319.

[2] Y. Lvet al., "Traffic Flow Prediction with Big Data: A DeepLearning Approach," IEEE Trans. Intelligent TransportationSystems, vol. 16, no. 2, 2015, pp. 865-73.

[3] Y. LeCun, Y. Bengio, and G. Hinton, "Deep Learning," Nature, vol. 521, no. 7553, 2015, pp. 436-44.

[4] Z. Liu et al., "Mining Road Network Correlation for TrafficEstimation via Compressive Sensing," IEEE Trans. IntelligentTransportation Systems, vol. 17, no. 7, 2016, pp. 1880-93.

[5] J. Zhang, Y. Zheng, and D. Qi, "Deep Spatio-Temporal Residual Networks for Citywide Crowd Flows Prediction," Proc. of AAAI, 2017, pp. 1655-61.

[6] J. Sun et al., "A Dynamic Bayesian Network Model for Real-Time Crash Prediction using Traffic Speed Conditions Data," Transportation Research Part C: Emerging Technologies, vol.54, 2015, pp. 176-86.

[7] X. Ma et al., "Learning Traffic as Images: A Deep ConvolutionalNeural Network for Large-Scale TransportationNetwork Speed Prediction," Sensors, vol. 17, no. 4, 2017, Article No. 818.

[8] J. Wang et al., "Traffic Speed Prediction and CongestionSource Exploration: A Deep Learning Method," Proc. IEEEICDM, 2016, pp. 499-508.

[9] ZhongjianLv et al "LC-RNN: A Deep Learning Model for Traffic Speed Prediction" Proc.Proceedings of the TwentySeventh International Joint Conference on Artificial Intelligence (IJCAI-18),pp 34703479 . 
[10] X. Mao et al., "Large-Scale Transportation Network CongestionEvolution Prediction using Deep Learning Theory,"PloS one, vol. 10, no. 3, 2015.

[11] R. Yu et al., "Deep Learning: A Generic Approach forExtreme Condition Traffic Forecasting," Proc. SIAM ICDM,2017, pp. 777-85.

[12] X. Song, H. Kanasugi, and R. Shibasaki, "DeepTransport: Prediction and Simulation of Human Mobility and TransportationMode at a Citywide Level," Proc. IJCAI, 2016, pp.2618-24.

[13] Q. Chen et al., "Learning Deep Representation from Bigand Heterogeneous Data for Traffic Accident Inference," Proc. AAAI, 2016, pp. 338-44.

[14] Y. Jia, J. Wu, and Y. Du, "Traffic Speed Prediction usingDeep Learning Method," Proc. IEEE ITSC, 2016, pp. 121722.

[15] X. Niu, Y. Zhu, and X. Zhang, "DeepSense: A Novel LearningMechanism for Traffic Prediction with Taxi GPS Traces," Proc. IEEE GLOBECOM, 2014, pp. 2745-50.

[16] M. Li et al., "Scaling Distributed Machine Learning with theParameter Server," Proc. USENIX OSDI, 2014, pp. 583-98

[17] Felix Kunde et al,'Traffic prediction using a Deep Learning Paradigm" proceedings of Joint Conference (March 21, 2017, Venice, Italy) on CEUR-WS.org (ISSN 1613-0073), pp301-309 march 2017.

[18] Thomas Epelbaumet al,"Deep Learning applied to Road Traffic Speed forecasting" Book, September 2017.

[19] Zhidan Liu et al,"Urban Traffic Prediction from Mobility Data Using Deep Learning" IEEE transactions on exploring deep learning for efficient and reliablemobile sensingNetwork ,pp 4046,Aug 2018. 